\title{
CORRIGENDUM
}

\section{Biochemical responses of broiler chicks to folate deficiency}

\author{
BY J. SARAH RENNIE, COLIN C. WHITEHEAD AND JOHN ARMSTRONG
}

Vol. 69 (1993), no. 3

Page 803 , Table 1 should be replaced by the following:

Table 1. Composition of basal experimental diet $(\mathrm{g} / \mathrm{kg})$

\begin{tabular}{lc}
\hline Casein (low-vitamin) & 186 \\
Gelatin & 94 \\
Wheat starch & 566 \\
Maize oil & 20 \\
Cellulose & 75 \\
Limestone flour & 20 \\
Dicalcium phosphate & 20 \\
Salt & 4 \\
L-arginine & $2 \cdot 7$ \\
L-tryptophan & $0 \cdot 13$ \\
L-cysteine & 0.52 \\
Vitamin and mineral supplement & 10 \\
\hline
\end{tabular}

* Provided $(\mathrm{mg} / \mathrm{kg}$ ) diet : copper $3 \cdot 5$, iodine $0 \cdot 4$, iron 80 , magnesium 300 , manganese 100 , zinc 50 , sodium molybdate 9 , sodium selenite 0.6 , potassium chloride $8 \mathrm{~g}$, retinol 2 , cholecalciferol $20 \mu \mathrm{g}, \alpha$-tocopherol 17 , menadione $1 \cdot 3$, thiamin 5 , riboflavin 4 , nicotinic acid 28 , pantothenic acid 10 , pyridoxine 8 , cyanocobalamin 0.01 , biotin $0 \cdot 3$, choline chloride $1.5 \mathrm{~g}$. 